\title{
Rachel Mapson \\ 6 Paths to politeness: Exploring how professional interpreters develop an understanding of politeness norms in British Sign Language and English
}

\begin{abstract}
This chapter explores how bimodal bilinguals acquire and develop their awareness of politeness in British Sign Language (BSL). Drawing on data collected through semi-structured group discussions involving eight highly experienced BSL/English interpreters the chapter focuses on how the participants learned about linguistic politeness in BSL and how this contrasts with their acquisition of English politeness norms. The data indicate how different paths to the acquisition of linguistic politeness might affect understanding of it. The experience of interpreters from Deaf family backgrounds, who acquired BSL as their first language, contrasts with those who learned BSL formally, as an additional language, as adults. Although both groups of participants acquired knowledge of politeness in similar arenas, the languages they were exposed to in these environments differed and intra-group experiences were heterogeneous. The data highlight the difficulty of learning politeness norms in an L2, with participants reporting a lack of explicit focus on politeness in BSL classes and interpreter training programmes. This may reflect the lack of literature on politeness in signed language, and on BSL in particular. Both groups of interpreters reported experiences involving the negative transfer of L1 politeness norms. Data indicate that the different modalities of BSL and English may facilitate transferability rather than restrict it, with one affordance being the 'blended transfer' of non-manual politeness features associated with BSL which may be performed simultaneously with spoken English.
\end{abstract}

Keywords: signed language, interpreters, bimodal bilinguals, cultural norms, pragmatic transfer, code-blending, blended transfer

\section{Introduction}

The extensive literature on linguistic politeness in spoken languages is not mirrored within sign linguistics, though existing studies on American Sign Language (Roush 1999, 2007; Hoza 2001, 2007), Japanese Sign Language (George 
2011) and British Sign Language (BSL; Mapson 2013) all concur that non-manual features form key politeness markers. These non-manual features relate to use of facial expression, particularly the mouth, eyes and brows, and to the use of the head and upper-body. Many of the cultural norms associated with BSL and other signed languages differ significantly from those of the dominant non-Deaf communities that surround them and these include consideration of (im)politeness. However, to date there has been little focus on how these politeness norms are acquired by native signers, or how these aspects of signed language are formally taught to L2 learners.

In spoken language research, the study of pragmatic development has been approached in a number of ways. Kasper (2001) summarises the four main perspectives involved as being: information processing, communicative competence, sociocultural and language socialisation. The latter two perspectives are frequently combined (Duff 2007) due to their complementary focus on assisted and experiential learning, and form a useful framework with which to consider the experiences of the interpreter participants involved in the present study. In contrast to the explicit instruction on L1 politeness norms provided by mothers to young children in Japan (Clancy 1986) and a continuing focus on explicit instruction within the pre-school environment there (Burdelski 2010), research suggests that an implicit approach is more prevalent in Western cultures (see Wolfson 1989; Snow et al. 1990; Blum-Kulka 1997; Kasper 2001). Lack of explicit instruction has also been identified within formal L2 teaching, though more explicit approaches are noted as beneficial (for example Thomas 1983; Béal 1994; House 1996; Jeon and Kaya 2006; Martínez-Flor and Alcón Soler 2007; Takahashi 2010; Woodfield and Economidou-Kogetsidis 2010). The distinction between pragmalinguistics and sociopragmatics (Leech 1983; Thomas 1983) may be relevant within the context of $\mathrm{L} 2$ tuition; though the two are interrelated (Béal 1994). Teaching the lexical and grammatical components of politeness (pragmalinguistics) is easier to effect in the classroom; Béal (1994) suggests that learners will readily correct such errors when they are pointed out. In contrast, the cultural and social norms (sociopragmatics) of the L2 are more difficult to convey in formal learning environments and L2 learners may be more resistant to adopting values that contrast with those of their L1 (Thomas 1983).

Research shows that the intersection of second language acquisition and pragmatics studies, described as 'interlanguage pragmatics' (e.g. Kasper and Blum-Kulka 1993; Kasper 1996; Bou Franch 1998), is a rich area of study. This chapter adds the additional layer of contrasting language modality to this discussion by examining the experiences of bilingual development of two languages in contrasting modalities; the aural/oral modality of English and the visual/spatial one of BSL. 
One manifestation of interlanguage pragmatics is pragmatic transfer. Like linguistic transfer more generally this relates to the transference of knowledge about one language into another. Sometimes knowledge can be successfully transferred (positive transfer) while on other occasions transference of L1 pragmatic knowledge into L2 is inappropriate (negative transfer). However, negative transfer does not necessarily result in 'pragmatic failure' (Leech 1983; Thomas 1983) as L1 speakers may make allowances for the lack of awareness in L2 use (Žegarac and Pennington 2008). Pragmatic transfer can be considered as either pragmalinguistic or sociopragmatic (Kasper 1992; Béal 1994) though these distinctions may be somewhat blurred (Žegarac and Pennington 2008).

The study of pragmatic transfer in sign/speech bilinguals adds a new perspective on the potential for both pragmalinguistic and sociopragmatic transfer. The present study looks at how this process relates to the acquisition of politeness in hearing bimodal bilinguals, exploring the experiences of those who acquired English as L1 and learned BSL formally as L2, and those with Deaf ${ }^{1}$ parents who acquired BSL as their L1 and English as L2. The latter group are referred to throughout this chapter as CODA (children of Deaf adults). First, I examine the literature relating to acquisition and formal learning of politeness and general issues concerning pragmatic transfer. I then relate this to the experiences of the professional sign language interpreters involved in my study and discussion around their understanding of politeness as being "about negotiation" and "the oil that enables us to live together peaceably." This chapter explores acquisition of politeness with particular focus on BSL, and the potential for negative pragmatic transfer. Their developmental journeys will be related to the order and circumstances in which their languages were acquired to explore the influence of the two languages on each another and highlight the heterogeneous nature of interpreters' experiences.

\section{Background}

\subsection{British Sign Language and the Deaf community}

It may be helpful to start by contextualising the use and transmission of BSL. BSL is the visual-gestural language used by the Deaf community in the United Kingdom. ${ }^{2}$ Brennan (1992) describes the strength of community members' identification with one another and their shared language, even though they are

1 The capitalised word Deaf is used to refer to deaf people who prefer to communicate in signed language and identify as members of a linguistic and cultural minority.

2 For further discussion about the membership and characteristics of the Deaf community see Kyle and Woll (1985). 
spread throughout the UK rather than residing in a discreet geographic area. BSL is totally independent from and unrelated to English. However, as English is the dominant majority language of the UK, it exerts considerable language contact influence on BSL (Sutton-Spence 1999); Deaf and non-Deaf (hearing) people interact with one another regularly and the majority of Deaf people have some knowledge of English.

In contrast with other linguistic minorities, transmission of language within the Deaf community is rarely from parent to child, and the language is more commonly acquired from peers, or other adults, at school. ${ }^{3}$ This is because an estimated 90 to 97 per cent of Deaf people are raised in non-Deaf families (Mitchell and Karchmer 2004).4 Though Deaf children may be exposed to English within home and school environments the language is not generally accessible to them in its spoken form, nor is written English always accessible to Deaf adults; there is evidence to suggest that the literacy skill of the majority of Deaf people is significantly lower than that of the general population (Conrad 1979; Powers, Gregory and Thoutenhoofd 1998). The communication barriers that Deaf people encounter with hearing people motivate their preference for socialising, using BSL, amongst their own community members. Deaf clubs, where BSL users gather socially, have traditionally been a focus for Deaf community members and have been recognised as an important setting for the transmission of their language and culture (Brennan 1992). The majority of Deaf parents have hearing children. These children, often known as CODAs, are one set of the bilinguals involved in the present study. Unlike most of their parents, these CODAs acquired BSL as their first language from their parents.

While the Deaf community may share some of the cultural norms of the majority population (Pietrosemoli 2001), they do not share all of them. This may stem from the inaccessibility of those norms and the different modalities of the languages involved; Deaf people operate in a visual and tactile world rather than one where sound and spoken language are often prioritised. These differences are exemplified in the cultural norms associated with turn-taking which in BSL is signalled through eye gaze ${ }^{5}$ (Sutton-Spence and Woll 1999). Cultural norms associated with attracting attention are also necessarily different from those generally used in spoken language, and physical touch for this purpose may be more common than within the British non-Deaf population.

3 Educational provision for Deaf pupils is now changing with a move towards educating Deaf children within mainstream education rather than at residential schools with other Deaf pupils. 4 Though this figure refers to data from the USA, it may be considered equally relevant to the UK, as percentages of genetically related deafness are the same within both populations.

5 Eye gaze in BSL has numerous other functions, as discussed in Sutton-Spence and Woll (1999). 
Differences relating to Deaf and hearing cultural norms associated with directness or indirectness have been discussed (e.g. Mindess 2006; Hoza 2007). These discussions suggest that direct requests that might be considered impolite by English-speakers and comments regarding personal appearance that hearing people would consider blunt and offensive might be judged very differently, and as culturally appropriate, by Deaf people.

One significant difference between signed and spoken languages is that the former makes use of the face and body in a number of ways that carry systematic meaning (Sutton-Spence and Woll 1999). This use of articulators other than the hands is referred to throughout this chapter as use of non-manual features. Nonmanual features are particularly relevant to politeness in signed language and have been acknowledged as key components of politeness in several signed languages (see Roush 1999, 2007; Hoza 2001, 2007; George 2011; Mapson 2013, 2014b). Mapson (2014b) indicates that lexical politeness markers, or manual signs, are non-essential for politeness in BSL as politeness can be conveyed entirely through non-manual features.

\subsection{Acquisition of politeness in $\mathrm{L} 1$}

Though the majority of research has been inclined towards the performance rather than the acquisition of pragmatic knowledge (Bardovi-Harlig 2001), Rose (2000) suggests that sociopragmatic development starts in the L1 of children by the age of around three years in contrast with a slower process of development of politeness norms for children learning an L2. Other studies indicate that ability to perform politeness continues to develop until around the age of eight (Ervin-Tripp and Gordon 1986; Ervin-Tripp, Guo and Lampert 1990) and Snow et al. (1990) suggest that this development continues through to adolescence. The generalised language socialisation processes described by Ochs (1996) as a means of children acquiring appropriate use of language, are related specifically to the development of 'pragmatic socialisation' by Blum-Kulka (1997) in her exploration of the communication and cultural behaviours of Israeli and Jewish-American families. Blum-Kulka's observations are based on dinner-table conversation, described as a rich environment for the development of pragmatic knowledge, presenting parents with the opportunity to model, correct and instruct children on language use. She relates her observation of family control styles to the work of Bernstein (1971) and his categorisation of the visible 'positional' control style and the more subtle 'personal' style; the former relates to the 'because I said so' mode of direction, while the latter involves a greater degree of negotiation. Blum-Kulka observes both styles during her own research and suggests that the 
style adopted is motivated both by cultural preferences and the purpose of the utterance, though middle-class parents demonstrated a preference for invisible 'personal' control. Snow et al. (1990) found that the language modelling of parents was similarly reflected in the language practices of extended family members. This assumes that all members of the extended family use the same language, which may not always be the case. For example, the vast majority of Deaf parents have non-Deaf (hearing) children, a figure estimated, as noted above, at between 90-97 per cent by Mitchell and Karchmer (2004). Similarly, most Deaf children are born to hearing parents, and therefore families where all members are Deaf are uncommon.

While the process of pragmatic acquisition is generally implicit (Snow et al. 1990; Kasper 2001), Blum-Kulka (1997) suggests that parents sometimes offer explicit instruction in the form of metapragmatic comments. These comments are made in a way that is reflective of the language culture and highlight the in/appropriateness of language use. How frequently comments are made may therefore relate to cultural norms. Snow et al. (1990) observed that even when explicit instruction on politeness was given by parents, this related mainly to what was appropriate in the context, and lacked any explication of the underlying 'rules' that inform appropriateness. It would therefore seem that, despite the complexity of politeness, children are largely left to deduce the rule system for themselves. Additionally, Blum-Kulka (1997) suggests that metapragmatic comments (e.g. a reminder to say 'please') are less common than metalinguistic ones that relate to language use in general, and relates this to a general lack of self-awareness about pragmatic issues, evidenced through observation and also in interviews with the parents. Similarly, Wolfson (1989) describes how the pragmatic knowledge of native speakers is usually implicit, rather than explicit, and therefore not easily described by them.

\subsection{Pragmatic development in L2}

Much of the literature on the teaching of pragmatics in L2 centres on the issue of implicit versus explicit tuition in formal learning situations. Taguchi (2011) describes explicit instruction as involving explanations about pragmatic features, alongside practice of them. While some authors indicate that pedagogical approaches may be better described as positioned along an implicit/explicit continuum (Jeon and Kaya 2006; Takahashi 2010) there is widespread agreement that explicit instruction is beneficial (e.g. Thomas 1983; House 1996; Martínez-Flor and Alcón Soler 2007; Taguchi 2011), facilitating the development of L2 pragmatic norms (Kasper 2001). Woodfield and Economidou-Kogetsidis 
(2010) suggest that this applies not only to those in the earlier stages of L2 development, but is also beneficial to advanced L2 users.

However, the explicit tuition of pragmatic norms is further problematised by a lack of codification on the subject, unlike grammar which may be taught on the basis of codified rules. The distinction between pragmalinguistics and sociopragmatics is useful in this regard. Of the two, pragmalinguistics is easier to convey in the classroom (Kasper and Rose 2001) where pragmalinguistic failure can be remedied with instruction about grammar and conventional language use (Thomas 1983). For example, Béal (1994) describes how French employees using English as an L2 in Australia can be made aware of common errors, such as the use of 'sorry' (transferred from their L1) when 'pardon' or 'excuse me' would be more appropriate in English. However, sociopragmatics is a more complex subject as it is far more dependent on subjective evaluations, more difficult to convey in a classroom setting (Kasper and Rose 2001). For example, the context-dependent element of these cultural expectations may create difficulty when teaching how imposition of a request may be perceived or how gratitude should be expressed appropriately. Adopting different cultural norms may be perceived by L2 learners as a challenge to their individual identity (Béal 1994) or their belief systems (Thomas 1983). Though pragmalinguistic correction may be readily accepted by the learner (Béal 1994), Thomas (1983) advocates that sociopragmatic errors should be identified and discussed rather than eliminated since learners may be more sensitive to issues concerning their social judgement. However, Béal (1994) indicates how pragmalinguistic errors may derive from sociopragmatic transfer, problematizing the distinction between these two categories, and both Thomas (1983) and Taguchi (2011) discuss how tutors may also consider correction of inappropriate politeness a sensitive issue, potentially resulting in students' ongoing misconceptions about language use.

One key component of the L2 learning experience within higher education establishments is the concept of the year abroad, during which students spend a prolonged period of residence amongst native speakers to facilitate experiential learning through interaction with native speakers. Understanding of L2 pragmatic norms develops in the process of interaction, and Shea (1994) discusses how learners are able to use the responses they get from native speakers during conversation to inform their own language development. Although study-abroad opportunities do not necessarily resolve all the difficulties in L2 pragmatic development (Bou Franch 1998; Kasper 1992), research conducted by Matsumura (2003) indicates that it is the amount of language exposure rather than the duration of stay in the L2 environment that is beneficial. These benefits are more difficult to achieve for L2 learners of a signed language. There is no 'country' for learners to visit and the potential for exposure is limited; Deaf signers form a linguistic 
minority dispersed within the general population, with current estimates of the Deaf population in the UK varying between $18,000-70,000 .^{6}$

The majority of studies have focused on the production of politeness in L2, rather than on the perception and judgement of it (Bardovi-Harlig 2001), but studies indicate that L1 speakers may perceive the politeness forms used by L2 learners in ways other than the speaker intends. Tanaka and Kawade (1982) indicate that ability to judge politeness is distinct from the ability to produce contextually appropriate politeness strategies, leading to Bardovi-Harlig's (2001) suggestion that this should be a focus of explicit instruction. She emphasises that linking these explications to context should be an integral part of this process. Lack of appreciation of how politeness may be perceived by others potentially leads to some of the issues identified by other researchers, such as the negative reactions to the misuse of 'please' by Japanese learners of English (White 1993). These problems may result from the reduced range of politeness strategies deployed by L2 learners (Tanaka and Kawade 1982; Thomas 1983), and noted by Rose (2000) specifically in relation to an over-reliance on politeness markers such as 'please' and 'thank you' in young L2 learners of English.

L2 learners may adopt the sociopragmatic norms of the L2 and/or rely on transfer of their L1 norms. Both Bardovi-Harlig (2001) and Taguchi (2011) indicate that, ultimately, the decision for a learner to adopt L2 sociopragmatic norms is a personal choice, recommending that these choices should be informed (Bardovi-Harlig 2001: 67) rather than unconscious actions resulting from lack of awareness (Thomas 1983; Taguchi 2011: 304).

\subsection{Pragmatic transfer}

The transfer of politeness norms from one language to another, whether done consciously or unconsciously, can lead to communication problems where interlocutors are unaware of cultural and linguistic differences which may affect how an L2 speaker is perceived (Kasper 1992). Greater understanding of pragmatic transfer can help with the identification and solution of problems in intercultural communication (Žegarac and Pennington 2008) and may therefore be an important consideration for those working as interpreters and translators.

\footnotetext{
6 Historically the number of Deaf BSL users in the UK has been estimated at between 50,000 and 70,000 (http://www.scod.org.uk/faqs/statistics/). However, the 2011 Census has generated some contradictory figures due to the differential wording of questions used in Scotland and England/Wales. The analysis of the Census data by the UK Council on Deafness now suggests a figure of 27,000 deaf BSL users in the UK (http://deafcouncil.org.uk/news/2013/11/18/2011census-data-on-number-of-bsl-users/). Sites accessed 31/07/2015.
} 
Bou Franch (1998) describes pragmatic transfer along a language/culture continuum, with pragmalinguistics and sociopragmatics at either end, but suggests that these two types cannot account for all pragmatic transfer. Bou Franch (1998) adopts the additional category of non-linguistic transfer proposed by Riley (1989) as a third type. This non-verbal form of transfer might relate to use of gestures, acceptable in one spoken-language culture but not in another, such as pointing with the index finger. However, categorising transfer as linguistic or non-linguistic may be problematic when this transfer occurs between languages in different modalities, and exemplifies potential differences between language production and perception. In Pietrosemoli's (2001) study of Deaf Venezuelans' use of politeness strategies when interacting with non-Deaf (hearing) people, she observes how Deaf people adopt gestures commonly used among the hearing population, in what she describes as a form of code-switching. One example is a sign that in Venezuelan Sign Language (LSV) means 'to die' but which, when used by hearing people has a far more colloquial meaning, comparable to 'kick the bucket.' These gestures are used by hearing people only in informal contexts and are considered inappropriate in situations involving a greater degree of formality or social distance, as is frequently the case in Deaf/hearing interactions. Pietrosemoli (2001) suggests that Deaf people's use of these borrowed signs is motivated by a concern for positive face maintenance with their hearing interlocutors. However, this intent is misunderstood by hearing people who perceive them as face-threatening gestures. This example of pragmatic transfer indicates that during bimodal interactions lexico-grammatical production in one language may be perceived as paralinguistic in the other. Here, the borrowing of hearing gestures results in negative sociopragmatic transfer.

The relationship between context and pragmatic transfer is discussed by Takahashi (2000), who indicates that familiarity with context may impact on an L2 speaker's thought processes making it easier for them to plan an utterance in L2. This research suggests that L2 utterances planned in L1 will exhibit greater L1 transfer. In contrast, those planned in L2 show a reduction of L1 transfer, a process that is facilitated by greater familiarity with the interactional context. Another perspective on L1/L2 influence is Cook's (2003: 10) comparison of bilingualism as akin to the operation of a mixer tap. He describes how both languages are always available to the speaker with the combination of the two languages adjusted according to context. It is therefore a question of how much of each of the two languages a speaker chooses to employ. This notion is an interesting one in relation to sign/speech bilinguals for two reasons. Firstly, because use of signed language may already exhibit some influence from the dominant spoken languages that surround them (Sutton-Spence and Woll 1999; Johnston and Schembri 2007), which may manifest in either the grammar or 
lexicon of the signed language. And secondly, because the constraints and affordances of bimodal bilingualism may affect the type and amount of transfer that occurs.

However, language transfer does not only occur from L1 into L2. Pragmatic transfer can also operate in the other direction, with L2 influencing L1 (BlumKulka 1991; Bou Franch 1998; Cenoz 2003; Pavlenko 2003). The bi-directional pragmatic transfer observed by Blum-Kulka (1997) included incidents of codemixing of L1/L2 at the lexical level, and code-switching at phrasal levels. Bou Franch (1998) describes 'transferability constraints' as conditions that promote or inhibit transfer. When considering pragmatic use in sign/speech bilinguals the bimodality of the language pair may actually afford less constraint in relation to transfer than might be expected, generating additional avenues of language transfer. For example, in a comparative study of sign/speech bilinguals and monolingual English speakers in the USA, Pyers and Emmorey (2008) found significant differences in the use of facial expressions associated with grammatical features in American Sign Language (ASL) during speech. The sign/ speech bilinguals involved in their study were all CODAs who had grown up with ASL as their L1. These participants made significantly more use of raised and furrowed brows in wh-questions and conditionals than the English-speaking monolinguals. Moreover, the timing of their use indicates they are being used in a grammatical way that carries over from their use in ASL in what is described as 'code-blending' (Pyers and Emmorey 2008). Where the monolingual English speakers used the same expressions, they did so for affect, with a wider range of idiosyncratic differences between them. While ASL and BSL are different languages, the use of brows as syntactic markers is common across sign languages (Zeshan 2004), and therefore a similar behaviour might therefore be anticipated in sign/speech bilinguals elsewhere.

\subsection{Summary}

The process of pragmatic acquisition is acknowledged as being predominantly implicit (Kasper 2001) and articulating this knowledge may therefore be difficult for research participants. There may be a relationship between the implicit nature of pragmatic socialisation and the lack of explicit instruction within formal teaching contexts. For L2 learners, this lack of explicit instruction may result in a lack of pragmatic understanding, and lead to a tendency to transfer cultural behaviours from L1 into the L2. The benefits of extended exposure to L2 cultural norms offered by study-abroad opportunities are not available to those studying signed languages, which may exacerbate the transfer of L1 sociopragmatic behaviours that are also problematic to address within formal tuition. 
Likewise, the degree of transferability (Bou Franch 1998) afforded to sign/speech bilinguals may influence the amount of linguistic transfer they produce. The present study examines participants' development of linguistic politeness and explores how the bimodal nature of their bilingualism impacts on bi-directional pragmatic transfer, reinforcing the discrepancies observed in earlier studies between the production and perception of linguistic politeness in intercultural contexts.

\section{Method}

\subsection{Participants}

Eight highly experienced BSL/English interpreters took part in the study; all come from white-British family backgrounds, are over 30 years of age, grew up in the UK and currently live in the South-East of England. These participants were divided into two groups shown in Table 1 and are referred to throughout this chapter by pseudonyms they selected for themselves. One group comprised four CODA interpreters from Deaf family backgrounds who acquired BSL naturally, as a first language, in early childhood. They also acquired English in childhood through exposure to hearing adults, siblings and peers in their home and community environments. Prior to the 1970s the vast majority of sign language interpreters were CODAs (Moody 2007) and they still represent a significant proportion, approximately 13 per cent, of those in the profession (Mapson 2014a). The other group are non-CODA participants who come from non-Deaf families and learned BSL formally, within adult education and university settings. Participant selection was not designed to be representative of the wider population.

Table 1: Participant groups

\begin{tabular}{ll}
\hline CODA & Non-CODA \\
\hline Jean & Emma \\
Pippi & Vivienne \\
Henry & Angus \\
Maurice & Olly \\
\hline
\end{tabular}

A purposive selection was made following pilot studies which indicated that more experienced interpreters would provide richer data as they would be able to draw on more extensive professional experience. Seven of the interpreters have worked as professional interpreters for more than 15 years, while the 
remaining participant has over ten years of experience. Interpreters with this level of experience represent approximately 30 per cent of the total BSL/English interpreters in the UK while over 50 per cent of interpreters have less than five years' of professional experience (Mapson 2014a). My own identity as a professional BSL/English interpreter assisted with the selection of suitable participants, and enabled me to recruit interpreters who knew one another and felt comfortable talking as a group.

\subsection{Data collection}

Data were generated in a series of semi-structured discussions with the two participant groups; pilot studies indicated that one session did not allow adequate time for in-depth discussion on such a complex topic. Each group therefore met on three occasions, with all sessions taking place within a six-month period at approximately two-monthly intervals, and sessions were scheduled at the convenience of the participants. Participants were aware that the topic of discussion was politeness in relation to their interpreting work, but no formal definition of linguistic politeness was given to them; they discussed their own notions of politeness and their personal experiences of it. Each discussion lasted just under two hours and included open questions regarding recognition of linguistic politeness in BSL and acquisition of politeness in BSL and English. In the second and third sessions these conversations were stimulated with a series of brief video clips of Deaf signers performing two speech acts commonly associated with politeness research, requests and apologies. Further discussion explored how these speech acts might be interpreted into spoken English and what contextual factors might influence their choices. Kasper (2000/2008) suggests that metapragmatic interviews such as this can be useful for initial explorations, as is the case in this study, particularly when a reflexive approach is involved. A semi-structured approach was adopted in order to ensure that key points were covered while facilitating the 'real conversation' described by Fontana and Frey (1998: 67). The familiarity already extant between group members encouraged lively debate and richer data generation than might have been achieved through individual interviews or in a more formal group situation.

Data were video recorded to capture richer data (Silverman 2000) including the use of both gesture and BSL. Often BSL signs were used alongside speech to support, exemplify or emphasise what was being spoken, while very occasionally they were used alone. The video recording additionally facilitated the transcription of the multiple voices involved, as pilot data exemplified how referencing to earlier comments is frequently made non-verbally. 


\subsection{Analysis}

Data were transcribed into written English and, whilst it is impossible to capture visual data entirely (Flewitt 2006), the transcription includes as much nonverbal detail as I perceived to be relevant to the discussion. These descriptions are written within square brackets following the text to which they refer. Likewise these brackets also incorporate transcription of BSL use represented in simplistic form, using a capitalised gloss, following the conventions described by Sutton-Spence and Woll (1999: xi).

Politeness, both in relation to BSL and to interpreting, is an under-researched topic. This study is therefore exploratory in nature and a thematic analysis was adopted as it is described as a valuable tool for this type of research (Braun and Clarke 2006). This approach allows for the incorporation of both theory-driven and data-driven coding; the latter is invaluable in a field where little theory exists. Initial coding was both theory and data-driven. This resulted in the creation of a thematic network (Attride-Stirling 2001) which allowed a visual representation of the data. The iterative process of data generation facilitated both inter and intra-group triangulation of the data. However, following the main discussion sessions, further time was spent with each of the participants to present back initial findings from the data and obtain their feedback.

\section{Discussion}

The data discussed here were generated during the first two sessions with each of the interpreter groups. Discussion included participants' reflections on learning about politeness, and their understanding of politeness in BSL. These data can be viewed from both sociocultural and language socialisation perspectives (Kasper 2001), and elements of each of these approaches will be drawn into this discussion. Although the present study is not the ethnographic model recommended by Kasper (2001) for the study of pragmatic development, the retrospective exploration about their experiences highlights participants' different journeys through pragmatic socialisation (Blum-Kulka 1997).

What emerged from the data was a sense of the different arenas in which knowledge of politeness was acquired and developed, namely: home, school, experiential learning, formal sign language tuition and interpreter training. Though these arenas were similar for both groups, there were inter-group differences in the general pattern of learning and a lack of homogeneity within intragroup experiences. The following discussion examines participants' paths to 
language acquisition and how this may relate to their knowledge and articulation of linguistic politeness in BSL. The data reveal differences in the way the CODA and non-CODA participants were able to articulate their knowledge of linguistic politeness in BSL, and in the depth of knowledge displayed. These differences may result from the different paths in their acquisition of linguistic politeness in BSL; CODA participants experienced bilingualism from early childhood, while for the non-CODA group the dual pathway didn't commence until adulthood. I then explore the various issues relating to pragmatic transfer experienced by all participants and how language transfer between BSL and English may be perceived by others.

\subsection{Acquisition of politeness as children}

Both groups described how their knowledge of politeness norms was acquired through their parents modelling the language, correcting their use of it, and sometimes explicitly instructing them on language use. Though the process was the same for each group, the language of the home was different; for the CODA group the language of the home was BSL, while for the other group the language was English.

Data from this study confirm previous observations that the implicit nature of pragmatic socialisation makes it a challenging topic for discussion; though the CODA participants were able to identify the pragmalinguistic features of BSL they were uncertain where or how that knowledge was acquired, as comment (1) indicates.

(1) "I remember being a little child and thinking is it please [PLEASE ending in closed hand] and thank you [THANK-YOU ending with open hand]. I remember inventing rules for myself.” (Henry)

Wolfson (1989) observed how, even with native speakers, knowledge about pragmatics is implicit rather than explicit and therefore not easily described; similarly Scollon and Scollon (2001) describe how the acquisition of cultural discourse mirrors the acquisition of language grammar and phonology. The CODA group discussion suggests that this tacit knowledge may be even harder to explicate when two languages and different cultural norms are involved as comments (2) and (3) indicate.

(2) "I think that I was taught how to be polite in this country, rather than about it being in English or BSL.” (Pippi) 
(3) "I think it's really hard to unpick what seems to be related to sign language per se and what relates to being a sign language user in England, or in a working class neighbourhood, or a middle class neighbourhood. There's so much overlap there that it's extremely difficult to work out what belongs to sign language.” (Maurice)

The difficulty in teasing apart tacit knowledge about politeness in both languages may relate in part to the language use within the extended family, most of whom would be non-Deaf, and the extent to which these family members were involved in the upbringing of the CODA participants. Despite this complexity, all CODA participants acknowledged their parents as being a source of pragmatic knowledge, especially in relation to polite requests and expressions of gratitude. Though implicit within much of the participants' discussion, they indicated that parents used metapragmatic comments such as those described by Blum-Kulka (1997) as a means of highlighting in/appropriateness. However, while dinner table interactions might provide a good introduction to conventional politeness in spoken languages, people using signed language are confronted with logistical issues which may influence interaction; it is not possible to hold a knife and fork and sign at the same time. So, for example, participants described leaning across the table to get items placed out of reach, or getting up in order to fetch something rather than ask for it to be passed to them which they would consider the norm with hearing people. Logistical considerations were also observed to influence parents' language use in other contexts; they would not, for example be able to thank a supermarket cashier in the same way that a hearing customer would. If a Deaf person is fiddling around with their purse they won't even have eye contact with the cashier, and CODA participants described how they automatically compensate for this when accompanying their parents by expressing gratitude on their behalf.

Styles of parental control varied, with both Pippi and Maurice describing strict home values that displayed a "Victorian" influence, equating with the more visible, or positional control styles described by Blum-Kulka (1997). For the CODA participants, a lack of group homogeneity is evident here in relation to the language use of extended family members, and this shows how consistency of language modelling by parents and extended family members (as observed by Snow et al. 1990) may not be a common experience for CODAs. For three participants their extended family were non-Deaf, or hearing, and they experienced a mix of BSL and English use within their home environment and early childhood socialisation. These bilingual experiences make it hard for participants to separate acquisition of one language from the other; alluding back to the metaphor created by Cook (2003) this struggle might be likened to 
the difficulty in separating hot and cold water that has been run through a mixer tap. However, the fourth participant had a rather different experience, again emphasising the lack of homogeneity within the CODA group.

(4) “I didn't have any hearing family members, like relatives, close by when I grew up, it was just the four of us, so for me anything to do with how hearing people behaved it was school. School was where I picked it up.” (Henry)

The two groups of participants had contrasting experiences of school. For the non-CODA group, school was very much a continuation of their learning about English politeness forms, though extending their socialisation into a more hierarchical setting in which interaction was more rule-bound and where particular forms of address were expected that were very different from communication at home. But the contrast with the home environment was much starker for the CODA group. For Henry, who grew up without the presence of any hearing extended family, attending school was his first exposure to "hearing behaviour."

Observation of appropriate behaviour and language was extended outside the home to experiential learning in early childhood interactions with others. For the CODA participants the home environment was not the only one where BSL politeness norms were acquired. Participants attended local Deaf Clubs with their parents, where their understanding of appropriate use of BSL developed further. These were social clubs that were attended regularly, often more than once a week, and were very much the heart of the Deaf community. Deaf clubs have now significantly declined in number and popularity, as opportunities for socialising have become more diverse (both face-to-face and online), but when the participants were growing up they were the key meeting place for Deaf people. Pragmatic socialisation occurred in these clubs, with participants describing how observation of interactions developed their knowledge about meaningful and appropriate language use. These clubs afforded Deaf people a rare opportunity to converse in BSL in a social setting outside the home. They were therefore an environment that allowed the CODAs to observe language use that varied from that of their parents due to the heterogeneous nature of the community, as exemplified in comment (5).

(5) “The Deaf world, in a sense, it's homogenous from the point of view that everybody is Deaf, but it is a very disparate group, you know particularly if you go to you know an event, let's say at the Deaf Club, where you've got Deaf people, maybe social workers right down to people who've never had a day's work in their lives and who can't read, and yet they are able to co-exist in that space." (Maurice) 
Participants appreciated the contrast between norms in BSL and English, with BSL characterised in terms of an involvement culture (Scollon and Scollon 2001; Mindess 2006). The participants discussed how intimacy within the Deaf community is much more immediate than it would be with non-Deaf people, but this degree of directness is not perceived as impolite. For example in (6) Henry describes how he is frequently greeted at the Deaf Club with a comment asking how his parents are, which contrasts with the conversational opener he would expect in a spoken interaction.

(6) "Well, a good example is when Deaf people meet up with me they'll say 'mother father well?' [he signs MOTHER FATHER WELL as he speaks] and you don't normally expect that kind of question at the very beginning of a conversation.” (Henry)

However, despite growing up as a member of the Deaf community, Henry described an underlying resentment to this style of greeting, later adding "well, I'll have your name first, then I'll tell you" which suggests that CODAs may experience some tension between the contrasting cultural norms of English and BSL.

\subsection{Experiential learning as adults}

Experiential learning about politeness continued for both groups into adulthood. The CODA participants described how they continued to learn about politeness in English through observation and interaction with others. However, they related how interactions with English speakers feel less natural for them, involving a degree of discomfort. Data suggest that the discomfort described when interacting with non-Deaf people may have deep psychological roots, which Maurice described as "an interplay between your innate personality and your persona as someone who occupies an English-speaking culture most of the time." Three participants revealed that they adopt a different persona when interacting in each of their two languages. For Henry and Maurice, this involved a more passive and introvert persona when speaking English; they described themselves as quiet and shy in this context rather than extrovert when communicating in BSL. Henry described himself as feeling "a bit swamped" within the hearing community, while Maurice described feeling he is "fencing" in these situations and forced into applying an un-natural set of rules. Interestingly, for the other participant this situation was reversed, again highlighting the heterogeneous nature of the group. Pippi described herself as being "much more outgoing and funny in spoken English than I am in BSL.” All of these comments, 
regardless of the distinctions expressed, perhaps suggest different perceptions of naturalness/markedness in the norms regulating the two languages.

All four non-CODA interpreters learned BSL as adults, and experiential learning at this stage was a key component of their learning experience. Their experiential learning ran in parallel to their formal tuition on the language, and again they described how observation of appropriate language use played a key part in their learning experience. However, the heterogeneous nature of individual experiences was again evident. Experiential learning as an adult was one participant's first exposure to BSL. Angus started learning about appropriate language use in BSL through saturation in a predominantly Deaf work environment, before commencing formal sign language tuition. In (7) he compared his work situation to a family environment, whereby his Deaf colleagues took on a parental role in correcting and instructing his language use.

(7) 'Kind of similar to when you're with your family, you know. And you're out on holiday and you just say something, in the wrong way, and then I'd be corrected. But sometimes quite harshly [mock sobbing]. But that was a good thing. Like in your family, you need to know, this is the wrong method, this is the right method." (Angus)

Angus went on to comment on how the motivation behind these corrections was to ensure he was using "the right behaviour for the Deaf world" suggesting that these comments related both to pragmalinguistic and sociopragmatic norms. He said he could "be really quite upset sometimes" for getting "the rules so badly wrong," reinforcing the sensitivity involved in shifting belief systems embedded in the sociopragmatic norms of L1 when learning an L2 (Thomas 1983). Thomas suggests that corrections about language are more easily received than those to do with social judgement. Angus added that fortunately his colleagues would not dwell on his mistakes, so having been "told off" he was able to recover his composure quickly. However, Angus's experiences were unique within the group. The other group members started learning BSL formally prior to, or in parallel with, socialising with Deaf people.

\subsection{Formal learning of BSL}

Discussion around participation in BSL classes and interpreter training indicates experiences of politeness being implicit within other topics rather than something that was explicitly taught. Like the non-CODA interpreters, three of the CODA group had some involvement in formal BSL tuition though for them the 
purpose was to ratify their language knowledge as a pre-requisite for formal interpreting qualifications rather than to learn the language. CODA participants recognised that politeness would have been addressed implicitly within curriculum topics such as register, language modification and in the way introductions are made within BSL culture. Similarly the non-CODA group reported that linguistic politeness was not explicitly taught. Formal BSL tuition was taught by Deaf tutors; so, while there was no explicit instruction on politeness, modelling of appropriate language would have occurred. Participants considered that these tutors may have been more reluctant to correct their students' mistakes, due to the nature of tutor/student interactions within higher education, experiences that resonate with Taguchi's findings (2011: 302). In comment (8), Olly contrasts his experiences of studying BSL at University with those at school, suggesting how the different relationships impacted on the giving of correction.

(8) "I think if you, even in an adult learning environment, you are rude or impolite, your adult instructors will respond to you quite differently to if you were a child and you're rude and impolite.” (Olly)

Though Kasper (2001: 520-521) suggests that explicit socialisation can occur in second language teaching, this appears not to have been the case for these participants. This may also reflect a more general lack of awareness and explicit knowledge about politeness in BSL in those who teach the language. The difficulties inherent in teaching sociopragmatics in particular (Kasper and Rose 2001) may be compounded by the deficit in literature on politeness in signed languages. BSL tutors' own implicit understanding and lack of awareness about politeness in BSL may influence how pragmalinguistics and sociopragmatics are taught. In addition, a dynamic involving tutors from a linguistic minority instructing students from the dominant language population may influence classroom interaction and further inhibit tutors from correcting mistakes. These issues add to the sensitivities involved in instigating changes to sociopragmatic behaviours (Thomas 1983) particularly as the classroom is not ideal for dealing with sociopragmatic knowledge (Kasper and Rose 2001).

The lack of explicit tuition about politeness in BSL may have been more problematic for those learning BSL as an L2, particularly as the same lack of explicit tuition was reported by both groups of participants in relation to interpreter training programmes. The formal training programmes undertaken by participants were all based in UK higher education establishments. While the issues covered within these training programmes included register, cultural mediation and how to reflect the intent of the speaker/signer, none of the participants in this study recalled any explicit discussion about politeness or how this 
could be conveyed in BSL. However, participants described how politeness would have been referred to implicitly within the detailed discussions about how BSL could be reflected appropriately in English. These discussions often related to video material of interactions in BSL and Olly described how students were asked "to look at not just what they're saying but how they're saying it, when they say it and when they pass turns.” Issues concerning eye-contact, turn-taking and the use of facial expression were frequently included in these analyses, particularly in relation to differences between formal and informal interactions.

(9) "I remember when there were video clips of Deaf people who they, themselves were introducing themselves to an audience, to give a presentation for example, how they did that, those opening seconds of getting people's attention, letting them know they were ready to begin, greeting them, introducing themselves, introducing the topic. The way it was done was broken down when we did practical work to produce our own English renditions of that material.” (Olly)

The CODA interpreters had already developed their knowledge of linguistic politeness in both BSL and English before they commenced interpreter training, and commented on the assumption that this knowledge was already developed for all students. Conversation about the difficulties that non-CODA interpreters might experience with this centred on the importance of developing this knowledge within social contexts rather than solely in formal tuition. Duration of exposure to BSL was perceived as important, with participants expressing concern that students who enter interpreting programmes with no prior knowledge of BSL would struggle to develop the pragmatic knowledge necessary for effective interpreting.

\subsection{Understanding politeness}

The different learning pathways of the two participant groups may be reflected in their understanding and articulation of issues relating to politeness in BSL. The non-CODA group learned BSL as adults, but the lack of explicit tuition on politeness became evident in the lack of detail they were able to articulate about the pragmalinguistic resources of that language. Discussion about polite BSL in this group was very hesitant, and although participants clearly appreciated that it is conveyed mainly through non-manual features, they were unforthcoming in providing more specific detail on this even when video prompts of BSL were used to stimulate discussion. 
It is possible that, as a result of the lack of formal input within the learning environment, the non-CODA participants based their understanding of politeness in BSL on their L1 knowledge. In comment (10), Vivienne succinctly makes this point.

(10) "I think when you're an adult you've got the groundings of politeness and manners and etiquette before you even learn a new language, whether that's Spanish or BSL or whatever. So you kind of think well, right, this is a new language but I know that if I do that or speak like that, that's rude, because you've got those rules already." (Vivienne)

This comment resonates with similar suggestions about sociopragmatic norms made by Thomas (1983) and Kasper (1992) and suggests an underlying assumption that politeness rules are universal. Participants indicated that direct observation of impoliteness was crucial in piecing together their understanding of politeness. Frequently, it was seeing impolite use of the language that helped them appreciate what could be considered polite. However there is some contradiction between Vivienne's suggestion of universal politeness rules in comment (10) and participants' acknowledgement of the differences between politeness in the two languages. This may result from differences between participants' awareness of pragmalinguistic resources and sociopragmatic norms. Because BSL and English co-exist in the same geographic area, L2 learners of BSL may be more inclined to consider sociopragmatic norms as being shared between the two languages. Alternatively, the participants may be describing the resistance towards L2 sociopragmatic norms described in earlier studies (Bardovi-Harlig 2001; Taguchi 2011).

The adult L2 learning and bilingualism of the non-CODA participants contrasts with the life-long bilingualism of the CODA group. The CODA participants were more articulate about their knowledge of linguistic politeness in BSL. However, the difficulties experienced by this group lay in the untangling of their understanding of politeness in BSL from their knowledge of English, and British culture. Though BSL is the L1 of these participants they also acquired English as children and grew up as bilinguals. During discussion they found it hard to separate knowledge of one language and culture from the other and it took time to unravel these issues.

CODA participants were able to draw on the benefit of this extended exposure to the two languages when articulating their knowledge of how politeness is performed in BSL. Their discussion generated spontaneous recognition of specific pragmalinguistic features in BSL, though they lacked the metalanguage to describe them, and therefore resorted to demonstration along with creative 
language use and metaphor to aid their descriptions. This is evident in Jean's description of the 'tight lips' mouth gesture, a pragmalinguistic feature used for mitigation in both BSL (Mapson 2013, 2014b) and ASL (Roush 1999, 2007; Hoza 2001, 2007).

(11) "I was thinking this week, and everything I could think of is all about facial expression. Like if you're interrupting someone. Maybe at most you'd be like [SORRY with slight polite duck and tight lips] but it's this like [points to her mouth] something going on there, just an apologetic mouth shape that comes out, that's kind of [tight lips] for the camera!” (Jean)

CODAs' knowledge of politeness in BSL was also reflected in discussions about the differences between the two languages, and in their recognition of the problems these differences sometimes generate within intercultural communication between Deaf and non-Deaf people.

(12) Henry: The way Deaf people ask a question, let's say if you're with a hearing person and they ask a hearing person a question, their face can sometimes come across as quite aggressive, but they're not aware of it, because of the inquisitive face like [questioning face, frown] 'what are you talking about?' but it's just not meant, it's meant like 'hmm, I'm just trying to make sense of what..'

Maurice: You wouldn't perceive that as impolite though?

Henry: Well, there's sometimes when it does feel like, 'tone the facial expression down a bit [with 'calm-down' gesture] because this hearing person won't ... you can see this person getting a bit [leans back] 'that's a bit rude!'

Henry's comments in this extended extract evidence his bicultural knowledge. While he understands the intent of the Deaf signer, he is also able to appreciate how that comment might be perceived negatively by an English speaker who may associate that facial expression with displays of anger.

\subsection{Language transfer}

The relationship between politeness in English and BSL was a recurring theme in the data for both groups of participants (as already shown in examples (2), 
(3) and (10)), and I now explore this issue of language transfer in more depth. The discussion focuses on transfer of sociopragmatic norms in relation to eye contact, the use of facial expression and lexical politeness markers, and concludes with an exploration of pragmatic transferability between the two languages.

CODA participants related how the sociopragmatic norms associated with eye-contact in BSL could be transferred inappropriately when interacting in spoken English, resulting in negative perceptions by their interlocutors. In comment (13), Maurice described the feedback he received from a teacher when he moved to high school.

(13) "The teacher I had, my class teacher in the first year, kind of took me on as a project, they knew my parents were Deaf. And he said something like, 'you look at people very intently.' As though it was a negative thing." (Maurice)

In BSL, maintaining eye contact with your interlocutor is a sociopragmatic norm and is a key indicator in the regulation of turn-taking. So while the need for eye contact might be considered a necessity due to the modality of the language it is also polite behaviour. When eye contact is not maintained this will be considered rude or indicate lack of interest and in effect is the equivalent of walking away in the middle of a spoken conversation. The non-CODA group appreciated the contrasting cultural norm relating to eye contact in English and BSL. Many British English speakers might consider the amount of eye contact essential for politeness in BSL quite intrusive. For those learning BSL as L2, avoidance of eye contact may be exacerbated by assumptions regarding universality of English cultural norms. Bou Franch (1998) suggests that sociopragmatic transfer is likely to happen when L2 speakers believe their L1 sociopragmatic norms to be universal, even though these assumptions may be mistaken (Kasper and Schmidt 1996). Assumptions regarding shared sociopragmatic norms for co-existing indigenous languages like BSL and English may be widespread. The different amount of eye contact required in polite BSL and English may therefore present something of a challenge to L2 learners of either language. Maurice's negative experience was far from unique and specific incidents were recalled by two other CODA interpreters who received critical feedback about their use of facial expression and body language. One of these was Jean who in (14) describes how the features used to convey politeness in BSL are frequently misinterpreted by non-signers. 
(14) "I remember once when I was 19, somebody said I should learn how to sort of put a mask on? Because I didn't realise that everybody could read everything on my face.” (Jean)

CODA participants' comments suggest that the 'volume' or intensity of facial expression used is greater than non-signers would expect, and may be perceived negatively during spoken interaction. This concurs with the experimental study conducted by Pyers and Emmorey (2008), who found that American CODAs use significantly more non-manual features during speech than non-signers; their use reflected the grammaticalised production of these features in ASL rather than idiosyncratic expression of affect. Though their research focused only on the use of raised and furrowed brows, data from the present study indicate that the grammaticalised use of other non-manual features may also occur during speech. These data, and those from the Pyers and Emmorey (2008) study, suggest that there may be some interesting discrepancies between production and perception. The comments of the CODA interpreters in the present study suggest that non-manual politeness markers (and the associated sociopragmatic norms relating to eye-contact in BSL) may be negatively transferred into English. This type of language transfer originates from the different modalities of the two languages in question. Some pragmalinguistic features of BSL can be produced concurrently with speech, and their use may be motivated by a desire to be polite. However, the same features may be perceived very differently by nonsigning English speakers. Further contrasts may exist between the production and perception of these features. Production of BSL pragmalinguistic markers during speech will not be perceived as linguistic transfer by non-signers, but simply as inappropriate facial expression. Similarly, use of BSL sociopragmatic politeness norms such as maintenance of eye-contact may not be perceived as relating to language. This relationship between how linguistic features are produced and the contrasting way they may be perceived extends the notion of non-linguistic pragmatic transfer described by Bou Franch (1998).

Another language transfer issue that emerged from the non-CODA discussion was the potential for inappropriate L1 transfer from English into BSL through the use of overly lexicalised forms of politeness. Those learning BSL as an L2 may be more inclined to use lexical forms of politeness such as the signs PLEASE and THANK-YOU, rather than using non-manual expression of politeness through use of facial expression and the upper body. Lexical politeness markers are non-essential in BSL as 'please' can be conveyed through a combination of non-manual politeness markers. However, the lack of explicit tuition identified earlier may result in L2 reliance on lexical markers, although as Vivienne illustrates in (14), the non-CODA participants recognised that polite signs such as 
PLEASE and THANK-YOU in BSL are used infrequently and that over-use of these signs is both un-necessary for politeness and may look strange.

(15) “For me it's not so much using the 'please' and 'thank yous,' that's almost too hearing, too English.” (Vivienne)

There is some resonance here with the perceived over-use of 'please' by Japanese L2 learners of English, generating unexpected responses from native-speakers (White 1993). The tendency for L2 learners of BSL to over-rely on signed lexicon rather than use of non-manual politeness markers may also reflect the over-use of specific politeness strategies for L2 learners in other languages (Rose 2000).

However, it is not only transfer from L1 that occurs. The non-CODA group reported what was described as "leakage" from their L2 into use of English.

(16) "I think there can be leakage. I probably do it, but I can certainly see it in other people who are interpreters. You see that, it spills. Some of those behaviours that are typically used when we're using sign language with Deaf people spill into your behaviour with people who are not Deaf.” (Olly)

For example, although identification of pragmalinguistic features in BSL was problematic for them, one participant demonstrated them while speaking about non-manual features generally. Some non-manual features can be produced concurrently with speech without interference to spoken English. This 'blended transfer' is possible due to the different modalities used by the two languages. This use mirrors the experience of L1 transfer described by the CODA group, which is again related to use of non-manual politeness markers and use of eyecontact. Though the blended nature of this transfer may be peculiar to sign/ speech bilinguals, the influence of L2 on L1 is not. Transfer of L2 use back into L1 has been noted in other studies (Blum-Kulka 1991; Cenoz 2003) and reflects the fluid nature of the L1/L2 relationship presented in Cook's (2003) 'mixer-tap metaphor.'

Data therefore suggest that the L1 transfer described by the CODA participants may be shared as L2 transfer by the non-CODA interpreters. Pyers and Emmorey (2008) suggest that complete inhibition of the non-selected (signed) language during speech is very difficult for sign/speech bilinguals to achieve. Constraints on transferability (Bou Franch 1998) are less restrictive for bimodal bilinguals, because elements of signed and spoken languages can be produced concurrently. This 'blended transfer' may occur when either manual signs or non-manual 
grammatical features are performed during speech and is a particular affordance of bimodal bilingualism.

\section{Conclusion}

The linguistic backgrounds of the two participant groups in this study, coupled with the bimodal nature of their bilingualism, present an interesting focus for exploration on the development of politeness. Though participants experienced different paths to their acquisition of politeness norms in their two working languages, the contexts associated with these learning opportunities were broadly similar. Both groups of participants found their experiences of politeness hard to articulate. For the CODA group this stemmed from their intuitive (and unconscious) knowledge and lifelong bilingual experience. For the non-CODA group it related to their lack of explicit learning about linguistic politeness in BSL. Neither group possessed the metalanguage with which to describe politeness in BSL, though the CODA participants were better able to compensate for this through demonstration and metaphorical description.

A lack of formal tuition on linguistic impoliteness was identified by all participants both in relation to BSL teaching and interpreter training programmes, although participants were able to identify implicit learning opportunities within these arenas. Participants' learning experiences reinforce earlier studies which highlight the sensitivities involved in the teaching of sociopragmatic behaviours (Thomas 1983). When sociopragmatic norms in the L2 contrast with those of the L1, as is the case with the un/acceptability of eye contact in English and BSL, this potentially creates difficulties, both for L2 learners of BSL and L2 English speakers, as a result of negative pragmatic transfer in either direction.

The data highlight the transferability (Bou Franch 1998) of pragmalinguistic features, as some pragmalinguistic features of BSL may be produced concurrently with speech in a form of blended transfer, a possibility afforded by the different language modalities. Participants' experiences suggest that these features may not always be perceived positively by non-signers. Furthermore, facial expressions used linguistically by a bimodal bilingual during speech may be perceived as non-linguistic by a monolingual speaker. These data therefore add to existing debates over the differences between production and perception of politeness as well as typology of pragmatic transfer. Additionally, the bi-directionality of transfer between BSL and English as both L1 and L2 provides further evidence for Cook's (2003) 'mixer tap' metaphor of the L1/L2 relationship in bilinguals.

The data discussed in this chapter were generated in a small-scale qualitative study with no intention of creating generalisations. As highly experienced 
interpreters, the participants do not represent the general BSL/English interpreting population within the UK. Pilot studies informed the selection of participants in order to facilitate the capture of richer data. Even so, the intuitive nature of participants' knowledge about linguistic politeness reinforces earlier comments on the difficulty of exploring the topic in interviews (Wolfson 1989; Blum-Kulka 1997). Data, even from such a small participant group, also reflects the heterogeneity of individuals' experiences. However, this study may form a useful foundation for further investigation in a number of areas. For example, the data in the present study usefully builds on the code-blending observed by Pyers and Emmorey (2008) in ASL/English bilinguals and one avenue could be a more detailed study into the blended transfer of linguistic politeness afforded to bi-modal bilinguals through the study of naturally occurring data.

\section{Acknowledgements}

I would like to thank all those who have participated in the various stages of my research, and to Rachel Sutton-Spence, Helen Woodfield, Barbara Pizziconi and Miriam Locher for their encouragement and the insightful comments they provided on earlier versions of this chapter.

\section{References}

Attride-Stirling, Jennifer. 2001. Thematic networks: An analytic tool for qualitative research. Qualitative Research 1(3). 385-405.

Bardovi-Harlig, Kathleen. 2001. Evaluating the empirical evidence: Grounds for instruction in pragmatics? In Kenneth R. Rose \& Gabriele Kasper (eds.), Pragmatics in language teaching (Cambridge Applied Linguistics Series), 13-32. Cambridge: Cambridge University Press.

Béal, Christine. 1994. Keeping the peace: A cross-cultural comparison of questions and requests in Australian English and French. Multilingua 13(1-2). 35-58.

Bernstein, Basil. 1971. Class, codes and control. Volume 1: Theoretical studies towards a sociology of language. London \& Boston: Routledge \& Kegan Paul.

Blum-Kulka, Shoshana. 1991. Interlanguage pragmatics: The case of requests. In Robert Phillipson, Eric Kellerman, Larry Selinker, Michael Sharwood Smith \& Merrill Swain (eds.), Foreign/ second language pedagogy research: A commemorative volume for Claus Faerch, 255-272. Clevedon: Multilingual Matters.

Blum-Kulka, Shoshana. 1997. Dinner talk: Cultural patterns of sociability and socialization in family discourse. Mahwah, N): Lawrence Erlbaum.

Bou Franch, Patricia. 1998. On pragmatic transfer. Studies in English Language and Linguistics 0. 5-20.

Braun, Virginia \& Victoria Clarke. 2006. Using thematic analysis in psychology. Qualitative Research in Psychology 3(2). 77-101. 
Brennan, Mary. 1992. The visual world of British sign language: An introduction. In David Brien (ed.), Dictionary of British sign language/English, 2-133. London: Faber \& Faber.

Burdelski, Matthew. 2010. Socializing politeness routines: Action, other-orientation, and embodiment in a Japanese preschool. Journal of Pragmatics 42(6). 1606-1621.

Cenoz, Jasone. 2003. The intercultural style hypothesis: L1 and L2 interaction in requesting behaviour. In Vivian Cook (ed.), Effects of the second language on the first (Second Language Acquisition 3), 62-80. Clevedon: Multilingual Matters.

Clancy, Patricia M. 1986. The acquisition of communicative style in Japanese. In Bambi B. Schieffelin \& Elinor Ochs (eds.), Language socialization across cultures (Studies in the Social and Cultural Foundations of Language 3), 213-250. Cambridge: Cambridge University Press.

Conrad, Reuben. 1979. The deaf schoolchild: Language and cognitive functioning. London: Harper \& Row.

Cook, Vivian. 2003. The changing L1 in the L2 user's mind. In Vivian Cook (ed.), Effects of the second language on the first (Second Language Acquisition 3), 1-18. Clevedon: Multilingual Matters.

Duff, Patricia A. 2007. Second language socialization as sociocultural theory: Insights and issues. Language Teaching 40(4). 309-319.

Ervin-Tripp, Susan \& David P. Gordon. 1986. The development of children's requests. In Richard L. Schiefelbusch (ed.), Communicative competence: Assessment and intervention, 61-96. San Diego, CA: College Hill Press.

Ervin-Tripp, Susan, Jiansheng Guo \& Martin Lampert. 1990. Politeness and persuasion in children's control acts. Journal of Pragmatics 14(2). 307-331.

Flewitt, Rosie. 2006. Using video to investigate preschool classroom interaction: Education research assumptions and methodological practices. Visual Communication 5(1). 25-50.

Fontana, Andrea \& James H. Frey. 1998. Interviewing: The art of science. In Norman K. Denzin \& Yvonna S. Lincoln (eds.), Collecting and interpreting qualitative materials 2nd edn., 47-78. London: Sage Publications.

George, Johnny Earl. 2011. Politeness in Japanese sign language (JSL): Polite JSL expression as evidence for intermodal language contact influence. Berkeley, CA: University of California doctoral dissertation.

House, Juliane. 1996. Developing pragmatic fluency in English as a foreign language: Routines and metapragmatic awareness. Studies in Second Language Acquisition 18(2). 225-252.

Hoza, Jack Eugene. 2001. The mitigation of face-threatening acts in interpreted interaction: Requests and rejections in American Sign Language and English. Boston: Boston University doctoral dissertation.

Hoza, Jack Eugene. 2007. It's not what you sign, it's how you sign it: Politeness in American sign language. Washington, DC: Gallaudet University Press.

Jeon, Eun Hee \& Tadayoshi Kaya. 2006. Effects of L2 instruction on interlanguage pragmatic development: A meta-analysis. In John M. Norris \& Lourdes Ortega (eds.), Synthesizing research on language learning and teaching (Language Learning \& Language Teaching 13), 165-211. Amsterdam: John Benjamins.

Johnston, Trevor \& Adam Schembri. 2007. Australian sign language (Auslan): An introduction to sign language linguistics. Cambridge: Cambridge University Press.

Kasper, Gabriele. 1992. Pragmatic transfer. Second Language Research 8(3). 203-231.

Kasper, Gabriele. 1996. Introduction: Interlanguage pragmatics in SLA. Studies in Second Language Acquisition 18. 145-148. 
Kasper, Gabriele. 2001. Four perspectives on L2 pragmatic development. Applied Linguistics 22(4). 502-530.

Kasper, Gabriele. 2008. Data collection in pragmatics research. In Helen Spencer-Oatey (ed.), Culturally speaking: Culture, communication and politeness theory 2nd edn., 279-301. London: Continuum.

Kasper, Gabriele and Shoshana Blum-Kulka (eds.) 1993. Interlanguage pragmatics. New York: Oxford University Press.

Kasper, Gabriele \& Kenneth R. Rose (eds.). 2001. Pragmatics in language teaching (Cambridge Applied Linguistics Series). Cambridge: Cambridge University Press.

Kasper, Gabriele \& Richard Schmidt. 1996. Developmental issues in interlanguage pragmatics. Studies in Second Language Acquisition 18(2). 149-170.

Kyle, Jim G. \& Bencie Woll. 1985. Sign language: The study of deaf people and their language. Cambridge: Cambridge University Press.

Leech, Geoffrey. 1983. Principles of pragmatics. New York: Longman.

Mapson, Rachel. 2013. Politeness in British sign language: the effects of language contact. In Alasdair N. Archibald (ed.), Multilingual theory and practice in applied linguistics: Proceedings of the 45th annual meeting of the British association for applied linguistics [BAAL], 167-170. London: Scitsiugnil Press.

Mapson, Rachel. 2014a. Who are we? Newsli 87. 13-15.

Mapson, Rachel. 2014b. Polite appearances: How non-manual features convey politeness in British sign language. Journal of Politeness Research 10(2). 157-184.

Martínez-Flor, Alicia \& Eva Alcón Soler. 2007. Developing pragmatic awareness of suggestions in the EFL classroom: A focus on instructional effects. Canadian Journal of Applied Linguistics 10(1). 47-76.

Matsumura, Shoichi. 2003. Modelling the relationships among interlanguage pragmatic development, L2 proficiency, and exposure to L2. Applied Linguistics 24(4). 465-491.

Mindess, Anna. 2006. Reading between the signs: Intercultural communication for sign language interpreters 2nd edn. Boston: Intercultural Press.

Mitchell, Ross E. \& Michael A. Karchmer. 2004. Chasing the mythical ten percent: Parental hearing status of deaf and hard of hearing students in the United States. Sign Language Studies 4(2). 138-163.

Moody, Bill. 2007. Literal vs. liberal: What is a faithful interpretation. The Sign Language Translator and Interpreter 1(2). 179-220.

Ochs, Elinor. 1996. Linguistic resources for socializing humanity. In John J. Gumperz \& Stephen C. Levinson (eds.), Rethinking linguistic relativity (Studies in the Social and Cultural Foundations of Language 17), 407-437. Cambridge: Cambridge University Press.

Pavlenko, Aneta. 2003. 'I feel clumsy speaking Russian': L2 influence on L1 in narratives of Russian L2 users of English. In Vivian Cook (ed.), Effects of the second language on the first (Second Language Acquisition 3), 32-61. Clevedon: Multilingual Matters.

Pietrosemoli, Lourdes. 2001. Politeness and Venezuelan sign language. In Valerie Dively, Melanie Metzger, Sarah Taub \& Anne Marie Bauer (eds.), Signed languages: Discoveries from international research, 63-79. Washington, DC: Gallaudet University Press.

Powers, Stephen, Susan Gregory \& Ernst D. Thoutenhoofd. 1998. The educational achievements of deaf children: A literature review. London: Department for Education \& Employment.

Pyers, Jennie E. \& Karen Emmorey. 2008. The face of bimodal bilingualism grammatical markers in American sign language are produced when bilinguals speak to English monolinguals. Psychological Science 19(6). 531-535. 
Riley, Philip. 1989. Well don't blame me! On the interpretation of pragmatic errors. In Wieslaw Oleksy (ed.), Contrastive pragmatics (Pragmatics \& Beyond New Series 3), 231-250. Amsterdam: John Benjamins.

Rose, Kenneth R. 2000. An exploratory cross-sectional study of interlanguage pragmatic development. Studies in Second Language Acquisition 22(1). 27-67.

Roush, Daniel. 1999. Indirectness strategies in American sign language: Requests and refusals. Washington, DC: Gallaudet University MA dissertation.

Roush, Daniel. 2007. Indirectness strategies in American Sign Language requests and refusals: Deconstructing the Deaf-as-Direct Stereotype. In Melanie Metzger and Earl Fleetwood (eds.), Translation, Sociolinguistic and Consumer Issues in Interpreting (Studies in Interpretation Volume 3), 103-156, Washington, DC: Gallaudet University Press.

Scollon, Ron \& Suzanne Wong Scollon. 2001. Intercultural communication: A discourse approach 2nd edn. Oxford: Blackwell

Shea, David P. 1994. Perspective and production: Structuring conversational participation across cultural borders. Pragmatics 4(3). 357-389.

Silverman, David. 2000. Doing qualitative research: A practical handbook. London: Sage Publications.

Snow, Catherine E., Rivka Y. Perlmann, Jean Berko Gleason \& Nahid Hooshyar. 1990. Developmental perspectives on politeness: Sources of children's knowledge. Journal of Pragmatics 14(2). 289-305.

Sutton-Spence, Rachel. 1999. The influence of English on British sign language. International Journal of Bilingualism 3(4). 363-394.

Sutton-Spence, Rachel \& Bencie Woll. 1999. The linguistics of British sign language: An introduction. Cambridge: Cambridge University Press.

Taguchi, Naoko. 2011. Teaching pragmatics: Trends and issues. Annual Review of Applied Linguistics 31. 289-310.

Takahashi, Satomi. 2000. Transfer in interlanguage pragmatics: New research agenda. Studies in Languages and Cultures 11. 109-128.

Takahashi, Satomi. 2010. Assessing learnability in second language pragmatics. In Anna Trosborg (ed.), Pragmatics across languages and cultures (Handbook of Pragmatics 7), 391-423. Berlin: Mouton de Gruyter.

Tanaka, Shigenori \& Saiki Kawade. 1982. Politeness strategies and second language acquisition. Studies in Second Language Acquisition 5(1). 18-33.

Thomas, Jenny. 1983. Cross-cultural pragmatic failure. Applied Linguistics 4(2). 91-112.

White, Ron. 1993. Saying please: Pragmalinguistic failure in English interaction. ELT Journal 47(3). 193-202.

Wolfson, Nessa. 1989. Perspectives: Sociolinguistics and TESOL. Cambridge, MA: Newbury House.

Woodfield, Helen \& Maria Economidou-Kogetsidis. 2010. 'I just need more time’: A study of native and non-native students' requests to faculty for an extension. Multilingua 29(1). 77-118.

Žegarac, Vladimir \& Martha C. Pennington. 2008. Pragmatic transfer. In Helen Spencer-Oatey (ed.), Culturally speaking: Culture, communication and politeness theory, 2nd edn., 141-163. London: Continuum.

Zeshan, Ulrike. 2004. Interrogative constructions in signed languages: Crosslinguistic perspectives. Language 80(1). 7-39. 